\title{
Inhibition of Microglial Activation Protects Hippocampal Neurogenesis and Improves Cognitive Deficits in a Transgenic Mouse Model for Alzheimer's Disease
}

\author{
Barbara Biscaro $^{\mathrm{a}, \mathrm{e}}$ Olle Lindvall ${ }^{\mathrm{b}, \mathrm{d}}$ Giuseppina Tesco ${ }^{\mathrm{e}}$ Christine T. Ekdahl $^{\mathrm{b}-\mathrm{d}}$ \\ Roger M. Nitsch ${ }^{\mathrm{a}}$ \\ ${ }^{a}$ Division of Psychiatry Research, University of Zurich, Zurich, Switzerland; ${ }^{b}$ Laboratory of Neurogenesis and \\ Cell Therapy, Wallenberg Neuroscience Center, ${ }^{C}$ Inflammation and Stem Cell Therapy Group, Division of Clinical \\ Neurophysiology, University Hospital, and ${ }^{d}$ Department of Clinical Sciences, Lund Stem Cell Center, Lund, Sweden; \\ 'Tufts University, Boston, Mass., USA
}

\section{Key Words}

Microglia $\cdot$ Minocycline $\cdot$ Amyloid precursor protein .

Presenilin $\cdot \beta$-Amyloid $\cdot$ Inflammation

\begin{abstract}
Background: Activated microglia with macrophage-like functions invade and surround $\beta$-amyloid $(A \beta)$ plaques in Alzheimer's disease $(A D)$, possibly contributing to the turnover of $A \beta$, but they can also secrete proinflammatory factors that may be involved in the pathogenesis of AD. Microglia are known to modulate adult hippocampal neurogenesis. $\mathbf{O b}$ jectives/Methods: To determine the role of microglia on neurogenesis in brains with $A \beta$ pathology, we inhibited microglial activation with the tetracycline derivative minocycline in doubly transgenic mice expressing mutant human amyloid precursor protein (APP) and mutant human presenilin-1 (PS1). Results: Minocycline increased the survival of new dentate granule cells in APP/PS1 mice indicated by more BrdU+/NeuN+ cells as compared to vehicle-treated transgenic littermates, accompanied by improved behavioral performance in a hippocampus-dependent learning task. Both brain levels of $A \beta$ and $A \beta$-related morphological deficits in the new neurons labeled with GFP-expressing retrovi-
\end{abstract}

\section{KARGER \\ Fax +4161306 1234 \\ E-Mail karger@karger.ch}

www.karger.com
(C) 2012 S. Karger AG, Basel

$1660-2854 / 12 / 0094-0187 \$ 38.00 / 0$

Accessible online at:

www.karger.com/ndd rus were unaffected in minocycline-treated mice. Conclusions: These results suggest a role for microglia in $A \beta$-related functional deficits and in suppressing the survival of new neurons, and show that modulation of microglial function with minocycline can protect hippocampal neurogenesis in the presence of $A \beta$ pathology.

Copyright $\odot 2012$ S. Karger AG, Basel

\section{Introduction}

Characteristic hallmarks of Alzheimer's disease (AD) include the deposition of $A \beta$, formation of neurofibrillary tangles, neurodegeneration along with signs of increased inflammatory responses $[1,2]$. Activated microglia and reactive astrocytes, often associated with $A \beta$ deposits, not only produce cytokines, chemokines, reactive oxygen species and neurotoxic factors, but may also contribute to neurotrophic activities [3-5]. In a transgenic mouse model of $\mathrm{AD}$ expressing amyloid precursor pro-

Roger M. Nitsch

Division of Psychiatry Research, University of Zurich

August Forel Strasse 1

CH-8008 Zurich (Switzerland)

E-Mail nitsch@bli.uzh.ch

Co-corresponding author:

Barbara Biscaro

Eisai Inc.

4 Corporate Drive, Andover, MA 01810 (USA)

E-Mail b.biscaro@gmail.com 
tein (APP)/mutant human presenilin-1 (PS1) mutations, microglia react to $A \beta$ pathology with a sequence of $d y-$ namic changes [6]. During the onset of $A \beta$ plaque deposition, activated CD11b+ (cluster of differentiation 11B, complement receptor, CR3, or Macrophage-1 antigen, MAC-1) microglia are present in the vicinity of $A \beta$ plaques, followed by increased expression of CD45 during progressing pathology, and by increased expression of MHC-II at late-stage of $A \beta$ pathology [4; B. Biscaro, unpubl. data]. However, experimental evidence suggests that neither the formation nor the maintenance of $A \beta$ or the associated neuritic dystrophy requires the presence of microglia in transgenic mice [7].

Neurogenesis, i.e. the generation of neurons from stem/progenitor cells, includes proliferation, fate specification, neuronal maturation and survival, and ultimately synaptic integration of newborn neurons into functional neuronal circuits $[8,9]$. In the adult mammalian brain, progenitor cells are located in the dentate gyrus subgranular zone (SGZ) of the hippocampus, as well as in the subventricular zone, lining the lateral ventricles. Proliferation and differentiation of neural stem cells are finely tuned by physiological stimuli, and neurogenesis is sensitive to pathological disease states affecting the microenvironment of the neural stem cell niches [9-11]. Glial cells influence these stem cell niches with a variety of secretory factors, and their effects on neurogenesis can be either damaging or supportive depending on the factors involved [12].

Minocycline is a tetracycline derivative with anti-inflammatory properties; it crosses the blood-brain barrier rapidly and inhibits microglia within the CNS. We have previously shown that minocycline restores neurogenesis after inflammation provoked by lipopolysaccharide infusion [13]. Minocycline is known to inhibit inflammation and microgliosis in several models of neurodegenerative disease including AD [14-19], but it is unknown how this relates to adult neurogenesis in the mouse models. The aim of the present study was to determine the action of microglia on neurogenesis in transgenic APP/ PS1 mice with pre-existing A $\beta$ pathology. While minocycline failed to affect reactive astrocytes, it reduced microglia in the dentate gyrus, decreased inducible nitric oxide synthase (iNOS) protein levels in brain homogenates as well as the reactivity of $A \beta$ plaque-associated $\mathrm{CD} 11 \mathrm{~b}+$ microglia in the hippocampus. Minocycline also increased the numbers of new neurons in the granule cell layer (GCL) without reducing cerebral A $\beta$. Downregulation of proinflammatory and upregulation of anti-inflammatory cytokines, as well as improved recognition memory, were associated with the minocycline administration to transgenic $\mathrm{AD}$ mice. Together, these results suggest that microglia are involved in the inhibition of adult neurogenesis during brain $\beta$-amyloidosis.

\section{Materials and Methods}

\section{Transgenic Mice}

Heterozygous doubly transgenic mice expressing both human mutant APP (Tg 2576) [20] and PS1 (mutation: M146L) were generated by crossing heterozygous APP (C57BL6/J $\times$ FVB/N $\times$ SJL/J background) with PS1 mice (Swiss Webster $\times$ B6D2F1 background). Genotypes were determined by PCR. Both males and females were housed in standard cages with females in groups of 2-4 and males single-caged under 12-hour light/dark conditions with ad libitum access to water and food. A total of 30 mice were included in the study (F1 generation, with the mixed background strain resulting from the breeding scheme described above), 15 of which were injected with GFP-expressing retroviral vector; $\mathrm{n}=5$ ( 3 females and 2 males) non-transgenic (non-tg) with vehicle treatment, which received retroviral vector infusion into the dentate gyrus; $\mathrm{n}=5$ ( 3 females and 2 males) non-tg with minocycline treatment; $\mathrm{n}=10$ ( 5 females and 5 males) APP/PS1 with vehicle treatment, of which 5 (3 females and 2 males) mice received retroviral vector infusion into the dentate gyrus; $\mathrm{n}=10$ (5 females and 5 males) APP/PS1 with minocycline treatment, of which 5 ( 2 females and 3 males) mice received retroviral vector infusion into the dentate gyrus. All experimental procedures followed guidelines set by the Swiss veterinary cantonal office for the use and care of laboratory animals (License No. 48/08).

\section{Minocycline Treatment}

Minocycline (Sigma-Aldrich, St. Louis, Mo., USA) was freshly prepared as a $5 \mathrm{mg} / \mathrm{ml}$ stock solution in dextrose $5 \%$ water (D5W, vehicle), pH 7.4, every 3 days. APP/PS1 mice as well as non-tg controls were 11 weeks old at the beginning of the experiment. APP/ PS1 received minocycline (50 $\mathrm{mg} / \mathrm{kg}$, i.p.), whereas control groups APP/PS1 and non-tg received vehicle $(10 \mu \mathrm{l} / \mathrm{g}$ body weight, i.p.) twice daily for the first 2 days and once daily for the next 5 days until stereotaxic surgery. Following retroviral vector injections, mice received halved dose of minocycline $(25 \mathrm{mg} / \mathrm{kg}$, i.p.) or vehicle once daily for the next 5 weeks. Animal body weights were measured weekly and the injection volumes were modified according to the detected changes. Two days before transcardial perfusion, all groups were subjected to behavioral testing as described below. Mice were perfused $24 \mathrm{~h}$ after the last injection and blood was withdrawn by heart puncture after deep anesthesia with ketamine/xylazine directly before perfusion, and separated from erythrocytes by centrifugation.

\section{Bromodeoxyuridine Labeling}

Starting the day of the retroviral vector infusion, mice were injected twice daily for three days with BrdU (50 mg/kg, i.p., dissolved in potassium-phosphate-buffered saline, KPBS) for labeling of mitotic cells [21]. Mice were sacrificed 5 weeks after the BrdU injections. 
Retrovirus-Mediated Labeling of New Hippocampal Neurons

An oncoretroviral vector derived from the Moloney sarcoma virus and expressing GFP under control of the Rous sarcoma virus promoter (MolRG) was used. The retroviral vector was produced and stereotactically injected into the dentate gyrus as previously described [22].

\section{Learning and Memory Assessments}

After 6 weeks' treatment with minocycline and 5 weeks after BrdU administration, all mice were moved to an inverted light cycle room 1 day after stereotaxic surgery and, on the day of the experiment, they were habituated to the testing room for at least $1 \mathrm{~h}$. Behavioral analysis was performed by an observer blinded to the experimental groups. Because the performance of mice subjected to retrovirus perfusion was comparable to that of mice that did not undergo surgery, the data were pooled within the same group of treatment.

Spatial Working Memory was assessed in a Y-maze task 2 days before perfusion. The total number of arm entries and the sequence of entries in the Y-maze were manually recorded during a 5 -min session. Data were analyzed to determine the number of arm entries without repetition. Success in this test is indicated by a high rate of alternation indicating that the animals can remember which arm was entered last. Results are presented as percentage of alternations, e.g. the ratio of actual divided with possible alternations (total number of arm entries -2) times 100. The performance of the animals in the maze was also digitally recorded with a computer-aided video analysis system (EthoVision, Noldus, Bayern, Germany).

Recognition Memory was tested in a hippocampus-dependent object recognition task [23]. On day 1 , mice were habituated to the empty testing cage (20 min). The testing cage was an opaque plastic chamber $(25 \times 40 \times 15 \mathrm{~cm})$ with bedding material from the home cages of the animals. In this way, mice in this olfactorysaturated environment extensively explore the objects rather than primarily examine the apparatus. On day 2 , the animals were set into the testing chamber now containing an object, and they were allowed to explore the object for $5 \mathrm{~min}$ ('familiarization phase'). After a 20-min delay, during which the mice returned to their home cages, the animals were replaced into the testing chamber that now contained the familiar object (object A) and a new object (object B) ('novelty phase'). The mice were given 5 min to explore the familiar and new objects during the second exposure. The familiarization and testing phases were filmed with a digital video camera for off-line analysis (EthoVision) and the times spent exploring familiar and novel object during the novelty phase were scored. 'Exploration' of objects A and/or B was defined as approaching the object nose-first within $2-4 \mathrm{~cm}$. A recognition index, calculated for each mouse, was expressed as the ratio (TB $\left.{ }^{*} 100\right) /(T A+T B)$, where $T A$ and $T B$ are the time spent during the second exposure on object $A$ and object $B$, respectively. All of the objects used were made of plastic or wood and did not differ in overall size but differed in shape and color. During the testing, 2 non-tg mice treated with minocycline had to be excluded because of stereotypic behavior, reducing the number of animals in this group to 3 .

\section{Tissue Preparation}

Mice that received retrovirus injection were anesthetized with ketamine/xylaxine, and were transcardially perfused with $50 \mathrm{ml}$
PBS, followed by $100 \mathrm{ml}$ of ice-cold $4 \%$ paraformaldehyde in $0.1 \mathrm{M}$ PBS, pH 7.4. Brains were removed, post-fixed overnight in the same medium, and put in $20 \%$ sucrose in $0.1 \mathrm{M}$ phosphate buffer for $24 \mathrm{~h}$. Systemically random coronal sections ( $40-\mu \mathrm{m}$ thick) were cut on a sliding microtome, with an interval of $320 \mu \mathrm{m}$ between consecutive sections, and stored in cryoprotective solution.

Mice that did not receive retrovirus injection were anesthetized with ketamine/xylaxine, and were transcardially perfused with $150 \mathrm{ml}$ of ice-cold PBS. Brains were removed and dissected through the midsagital plane. One hemisphere was either further dissected into cortex and hippocampus and snap-frozen in liquid nitrogen and stored at $-80^{\circ} \mathrm{C}$, or fixed with $4 \%$ paraformaldehyde for $24 \mathrm{~h}$ at $4^{\circ} \mathrm{C}$ and processed as described above.

\section{Protein Extraction}

Cortices and hippocampi were homogenized in 10 tissue volumes (w/v) of $50 \mathrm{mM}$ Tris-HCl, $150 \mathrm{mM} \mathrm{NaCl}, \mathrm{pH} 7.5$, containing complete protease inhibitor cocktail (Roche, Basel, Switzerland) with Teflon-glass homogenizer (40 strokes) and subsequently centrifuged at $14,000 \mathrm{~g}$ for $50 \mathrm{~min}$ at $4{ }^{\circ} \mathrm{C}$ (soluble fraction). The supernatants were collected, and, prior to storage at $-80^{\circ} \mathrm{C}$, the protein concentrations were determined using the BCA Protein Assay (Pierce Biotechnology, Rockford, Ill., USA). Pellets were further processed with $70 \%$ formic acid (FA) to solubilize A $\beta$ from the aggregated $A \beta$ plaques, and spun at $100,000 \mathrm{~g}$ for $1 \mathrm{~h}$ at $8{ }^{\circ} \mathrm{C}$ (insoluble fraction). Aliquots of the $70 \%$ FA extraction supernatant were lyophilized overnight in order to remove the acid, and stored at $-80^{\circ} \mathrm{C}$. Because at 4 months of age the accumulation of $\mathrm{A} \beta$ in the brain of APP/PS1 mice is most prominent in the cortex, extracts from cortices were used to evaluate $A \beta$ and iNOS concentrations, whereas extracts from hippocampi were used to evaluate cytokine concentrations in the vicinity of the neurogenic niches.

\section{Immunohistochemistry}

For epitope retrieval of BrdU and of the proliferating cell nuclear antigen (PCNA) (specifically expressed in early G1 and S phases of the cell cycle, thus identifying the actual number of dividing cells [24]), free-floating sections were denatured in $1 \mathrm{M} \mathrm{HCl}$ for $30 \mathrm{~min}$ at $+60^{\circ} \mathrm{C}$. Preincubation with $5 \%$ appropriate normal sera in KPBS containing $0.25 \%$ Triton-X-100 (T-KPBS) was carried out for $1 \mathrm{~h}$ at room temperature. Primary antibodies were: rabbit anti-ionized calcium-binding adapter molecule 1 (Iba1) (a marker for microglia/macrophages, [25], 1:1,000, Wako Chemicals, Osaka, Japan); mouse anti-human A $\beta$ (6E10) (1:1,000, Covance, Dedham, Mass., USA); rat anti-CD11b (CD11b expression is up-regulated in activated microglia, 1:200, BD Pharmingen, San Jose, Calif., USA); guinea pig anti-glial fibrillary acidic protein (GFAP) (for identification of astrocytes, 1:1,000, Advanced Immunochemicals, Long Beach, Calif., USA); rat anti-BrdU (1:100, Oxford Biotec, Oxfordshire, UK); mouse anti-neuronal nuclei marker (NeuN) (1:100, Chemicon, Temecula, Calif., USA); rabbit anti-S100 $\beta$ (a marker for astrocytes; 1:5,000, SWANT, Bellinzona, Switzerland); goat anti-doublecortin (a marker for immature neurons; 1:400, Santa Cruz Biotechnology, Santa Cruz, Calif., USA), rabbit anti-Ki-67 (a marker for proliferating cells in G1, S and G2 phases; 1:500, Biocare Medical, Concord, Calif., USA). Free-floating sections were incubated with the primary antibody overnight at $+4^{\circ} \mathrm{C}$, in T-KPBS with $2 \%$ appropriate normal sera. Secondary antibodies for detection were Cy3-conjugated 
donkey anti-rabbit/rat, FITC-conjugated goat anti-mouse/rabbit, Cy5-conjugated donkey anti-mouse/guinea pig with incubation for $2 \mathrm{~h}$ at room temperature in the dark. Rinsing in T-KPBS was carried out between the incubations. For single-staining with mouse anti-PCNA (1:200, Santa Cruz Biotec), avidin-biotin-peroxidase complex (Elite ABC kit, Vector Laboratories, Burlingame, Calif., USA), 3,3'-diaminobenzidine and hydrogen peroxide were used. Chromogenic visualization included pretreatment with blocking of endogenous peroxidase activity with $3 \% \mathrm{H}_{2} \mathrm{O}_{2}$ and $10 \%$ methanol. Sections were mounted on chrom-gelatin-coated microscope slides (Super-frost-plus; Menzel, Braunschweig, Germany) and coverslipped with aqueous anti-fading mounting medium (PVA-DABCO). Thioflavin-S (ThioS) staining for fibrillar $A \beta$ was performed as described previously [26]. Sections stained with ThioS were sequentially stained with $6 \mathrm{E} 10$ to detect the remaining, not-compact $A \beta$ deposits. Control stainings included omission of the primary antibody and/or use of different tissues known to be positive for the specific antibody.

\section{Microscopic Analyses and Stereology}

All measurements were performed by an observer blind to animal identifications. Immunohistochemical stainings were examined with an Inverted Leica DM IRE2 fluorescence and light microscope. Numbers of immunoreactive cells were counted in 4-5 consecutive sections, $320 \mu \mathrm{m}$ apart from each throughout the hippocampus (DV: -1.8 to -3.0 ) in the GCL, and within a 1-cell diameter below this region in the SGZ. Stereological estimations of the total number of BrdU+ in the SGZ/GCL as well as numbers of Iba1+ microglia and GFAP+ astrocytes in the dentate gyrus (GCL/SGZ + hilus) were performed using the optical fractionator method $[27,28]$ with the support of newCAST software (Visiopharm, Copenhagen, Denmark) connected to a Leica DM4000B microscope $(\times 100$ oil objective $)$ with an Olympus DP71 color digital camera. For systematic sampling, the frame area was set to $3,590 \mu \mathrm{m}^{2}$ with a sampling interval of $226 \mu \mathrm{m}$ at the $\mathrm{x}$ and $\mathrm{y}$ lev$\mathrm{el}$, and the optical disector constituting a $15-\mu \mathrm{m}$-thick fraction of the total section thickness (measuring an average of 25-27 $\mu \mathrm{m}$ after processing). Neither the area for counting nor the thickness of the analyzed sections differed between groups, which allowed for comparisons of cell counts. Colocalization of up to 50 doublestained BrdU+ cells with NeuN or S100 $\beta$ as well as PCNA+ with $\mathrm{DCX}+$ cells was validated using a confocal scanning microscope (Leica TCS/SP2, Leica, Wetzlar, Germany) with argon laser 488, HeNE laser 568 and 633 excitation filters. Because CD11b was detectable only surrounding the $A \beta$ plaques, its immunoreactivity was evaluated by image analysis in the whole hippocampus (see below) rather than by cell counting due to the broader area considered and the spot-like appearance. A $\beta$ deposition was also evaluated by image analysis of cortices and hippocampi, as described below.

\section{Morphological Analysis of New GFP+ Mature Neurons}

Detailed description of this analysis is provided elsewhere [22]. Briefly, the dendritic lengths and branching of 5-week-old $\mathrm{GFP}+$ new granular cells ( $\mathrm{n}=10$ per group) were analyzed by using the Leica DM4000 microscope with a $\times 20$ water objective and a digital zoom of $2(1,024 \times 1,024$ pixels). For spine density analysis, pictures of 2-3 dendritic segments $40 \mu \mathrm{m}$ long $(n=25$ segments per group) per GFP+ cell analyzed were also taken using the Leica DM4000 microscope with a $\times 63$ water objective and a digital zoom of $6(512 \times 512$ pixels $)$. The fluorescence intensity of the labeled neurons was such that anti-GFP staining was unnecessary. After deconvolution using the open source Huygens remote manager (http://hrm.sourceforge.net/), 3D reconstructions of confocal images were performed with Imaris 6.1 (Bitplane, $\mathrm{Zu}$ rich, Switzerland). After 3D reconstructions, Scholl analysis and spine classification (stubby, long-thin and mushroom) were done using the aforementioned software and its MatLab extensions (ImarisXT).

\section{Image Analysis and Microglia Morphology}

Before measuring areas with ImageJ (http://rsb.info.nih.gov/ ij/), all images were subjected to the following transformations: color pictures were first converted to grayscale images (8-bit) and then the resulting images were further adjusted to black and white by defining a grayscale cutoff point (threshold). All pictures were adjusted to the same threshold within the group of study, to allow comparison of the results. Quantification of diffuse plaque load (6E10 staining), compact plaque burden (ThioS), and activated microglia (CD11b) was reported as area fraction (\% area), defined as the area positive for the staining in a constant and determined area of the cortex and hippocampus. The morphology of Iba1+ microglia in the dentate gyrus and septum was characterized as ramified, intermediate, amoeboid or round profile, as described elsewhere [29], and the percentage of the respective phenotype was calculated over the total number of Iba1+ cells considered (approximately 50 cells).

\section{Measurements of Mouse Cytokines}

All samples were normalized to $400 \mu \mathrm{g} / \mathrm{ml}$ before analysis. Levels of the pro-inflammatory cytokines interleukin-6 (IL-6) and tumor necrosis factor- $\alpha$ (TNF- $\alpha$ ) as well as level of the antiinflammatory IL-10 in hippocampal supernatants and plasma were measured using ELISA kits per manufacturer's instructions (Invitrogen, Basel, Switzerland). Cytokines concentrations were normalized to total hippocampal protein concentration.

\section{Western Blotting}

Tissue extracts were separated by SDS-PAGE using 10-20\% Tricine gels (Invitrogen) and transferred onto nitrocellulose membranes. After an optional epitope retrieval and blocking in $5 \%$ milk in Tris-buffered saline with $0.01 \%$ Tween-20, the membranes were probed with biotinylated-6E10 (1:500) antibody, and with rabbit anti-iNOS (1:1,000, Millipore, Billerica, Mass., USA). Secondary antibody was streptavidin-conjugated peroxidase or anti-rabbit peroxidase (1:10,000, Jackson Laboratories, Ben Harbor, ME). Immunopositive bands were visualized by chemiluminescence (ECL, Amersham Biosciences) and subsequently quantified by densitometric analysis of their intensity levels. $\beta$-Actin or GAPDH were used as loading control (1:10,000, Sigma, St. Louis, Mo., USA). The results were expressed as signal intensities normalized to the respective $\beta$-actin or GAPDH signals.

\section{$A \beta_{40}$ and $A \beta_{42}$ ELISA of Cerebral $A \beta$}

The $A \beta_{40}$ and $A \beta_{42}$ quantities in the cortical soluble and insoluble fractions were determined using $\mathrm{hA} \beta_{40}$ and $\mathrm{hA} \beta_{42}$ ELISA kits according to the manufacturer's instructions (The Genetics Company AG, Basel, Switzerland). Soluble fractions were normalized to total cortical protein concentrations. 
Statistical Analyses

Statistical differences between groups were detected using, when appropriate, either one-way ANOVA followed by Bonferroni post hoc test or the non-parametric Kruskal-Wallis test, followed by pairwise comparisons using the Mann-Whitney U test. Spearman's rank correlation coefficient (rho) was calculated to measure the statistical dependence of two variables. Data are presented as mean \pm SEM. Differences are considered significant at $\mathrm{p}<0.05$. Calculations were made with Statview 5.0.

\section{Results}

\section{Minocycline Reduces Microglia Activation in the}

Brain of APP/PS1 Mice

The doubly transgenic APP/PS1 mice used in our experiments started to deposit $A \beta$ plaques in cerebral cortex and the CA1 region of the hippocampus at 2-3 months of age, consistent with previous findings [30]. At 4 months of age, these mice exhibited compact $A \beta$ plaques evenly scattered throughout the cortex and the outer molecular layer of the hippocampus, with approximately $1 \%$ of brain sections covered with $A \beta$. Treatment of 3-monthold mice with minocycline for 6 weeks reduced the numbers of Iba + microglia in the dentate gyrus by about $43 \%$, attaining numbers of age-matched non-tg mice (fig. 1a, g, k). Merged overlays of triple-stained hippocampi show total microglia (Ibal - green), intermediate- and amoeboid-like microglia over-expressing CD11b (CD11b - red) and astrocytes (GFAP - blue) in control-treated and minocycline-treated APP/PS1 mice (fig. 1j, n, respectively). We observed that remaining CD11b+ microglia were only present in the vicinity of $A \beta$ deposits. Minocycline reduced the CD11b-immunoreactivity (IR) in these cells (fig. $1 \mathrm{~b}, \mathrm{~h}, \mathrm{l}$ ) in the hippocampus. A decline also seemed to occur in the cortical areas, but it did not reach statistical significance (APP/PS1: $0.07 \pm 0.03 \mathrm{APP} / \mathrm{PS} 1+$ minocycline: $0.02 \pm 0.01 \%$ area, $\mathrm{p}=0.17)$. Minocycline normalized the increased hippocampal levels of the proinflammatory cytokines IL- 6 and TNF- $\alpha$ (fig. 1d, e). Interestingly, these decreases were associated with a trend towards increased IL-10, an anti-inflammatory cytokine that can inhibit the synthesis of proinflammatory cytokines (fig. 1f). Levels of IL-6 strongly correlated with the numbers of Iba1+ microglia, both in minocycline- and control-treated APP/PS1 mice (fig. 1c, rho $=0.88$ ). Plasma levels of these cytokines were below detection limits. At 3 months of age, microglia did not yet express MHC-II; MHC-II was detectable, however, in 11- to 12-month-old mice with more advanced $A \beta$ pathology. Treatment with minocycline did not alter the activation state of the
Table 1. Relative frequencies (in \%) of morphological phenotypes of Iba1+ microglia in non-tg and APP/PS1 doubly transgenic mice subjected to minocycline or vehicle administration

\begin{tabular}{lcccc}
\hline & Ramified & $\begin{array}{l}\text { Inter- } \\
\text { mediate }\end{array}$ & $\begin{array}{l}\text { Amoe- } \\
\text { boid }\end{array}$ & Round \\
\hline Non-tg & $83 \pm 5$ & $16 \pm 5$ & 0 & 0 \\
Non-tg + minocycline & $80 \pm 6$ & $19 \pm 6$ & $0.7 \pm 0.7$ & 0 \\
APP/PS1 & $66 \pm 7$ & $27 \pm 4$ & $7 \pm 5$ & 0 \\
APP/PS1 + minocycline & $83 \pm 7$ & $15 \pm 6$ & $2 \pm 1$ & 0 \\
\hline
\end{tabular}

Morphological subtypes of Iba1+ microglia: ramified and intermediate represent quiescent and amoeboid and round represent activated microglia. No significant differences were observed between groups. Values are means + SEM; $\mathrm{n}=5$ per group.

remaining microglia as indicated by the unchanged relative frequencies of ramified, intermediate, amoeboid, and round Ibal+ cells (table 1). Minocycline did not alter the numbers of GFAP+ astrocytes in the dentate gyrus (fig. 1i, $\mathrm{m}$, and online supplementary figure $\mathrm{S1}$, www. karger.com/doi/10.1159/000330363).

The iNOS releases NO solely in response to inflammation triggers. In the brain, iNOS is mainly induced in glia around sites of inflammation, e.g. A $\beta$ plaques, and the production and release of NO by iNOS is associated with neuronal damage [31]. Notably, following minocycline treatment the levels of iNOS in cortical homogenates were significantly reduced in APP/PS1 mice as compared to vehicle-treated APP/PS1 littermates, while the levels of iNOS in non-tg controls were below detection (fig. 1o, p). We were unable to detect differences in the levels of gliosis within the same treatment group associated with the stereotactic injections.

\section{Minocycline Protects Neurogenesis in the Absence of}

Reductions in $A \beta$ Plaques

While blockade with minocycline of acute inflammation following lipopolysaccharide challenge improves the survival of newly born neurons $[13,32]$, the effects of minocycline on neurogenesis during the chronic neuroinflammation related to $A \beta$ pathology are unknown. In 3 -month-old doubly transgenic mice, minocycline treatment increased the numbers of $\mathrm{BrdU}+/ \mathrm{NeuN}+$ mature neurons by $45 \%$ as compared to littermates that received vehicle ( $\mathrm{p}=0.0061$; fig. 2 ) and had significantly less $\mathrm{BrdU}+/ \mathrm{NeuN}+$ cells than non-tg controls $(\mathrm{p}=0.0014)$.

Proliferating astrocytes, measured as numbers of $\mathrm{BrdU}+/ \mathrm{S} 100 \beta+$ cells, were not detectable in non-tg ani- 
Fig. 1. a, b Minocycline treatment reduces microglia activation in APP/PS1 mice. The treatment reduced the numbers of Iba+ microglia in the dentate gyrus of APP/ PS1 mice to non-tg controls levels as well as decreased CD11b-immunoreactivity (IR) in the hippocampus of APP/PS1 mice. d, e Minocycline did not significantly reduce the levels of TNF- $\alpha$, but downregulated the concentrations of IL-6 $(\mathrm{p}=0.06)$. f The anti-inflammatory treatment was also associated with higher levels of the anti-inflammatory cytokine IL-10 ( $\mathrm{p}=0.07)$, ( $\mathrm{n}=5$ per group/cytokine). $\mathrm{c}$ The number of Iba1+ microglia positively correlated with levels of IL-6. g-n Photomicrographs of immunofluorescent triple-labeled hippocampi show total microglia (Ibal green), activated microglia (CD11b - red), astrocytes (GFAP - blue) and respective overlay for APP/PS1 (g-j), and for APP/ PS1 minocycline-treated mice (k-n). Insets in $\mathbf{h}$ and $\mathbf{I}$ represent high-magnification details of CD11b+ microglia selected by the yellow squares. 0 Western blot analysis of cortical samples to measure the levels of inflammation-driven protein iNOS in non-tg and APP/PS1 animals (middle lane). The same blot was then reprobed with anti- $\beta$ amyloid antibody 6 E10 to confirm the presence of the human APP transgene in these samples (upper lane). Equal protein loadings were confirmed by measuring GAPDH (lower lane). There was a significant decrease in densitometric values of iNOS protein in brain homogenates of APP/PS1 mice treated with minocycline. Scale bars $=60 \mu \mathrm{m}(\mathbf{g}-\mathbf{n})$ and $5 \mu \mathrm{m}$ (insets). Dashed lines depict the SGZ. $\mathrm{OML}=$ Outer molecular layer. Error bars represent SEM. * $\mathrm{p}<0.05,{ }^{*} \mathrm{p}<0.01$, ANOVA followed by Bonferroni post hoc test, Mann-Whitney U test and Spearman correlation.

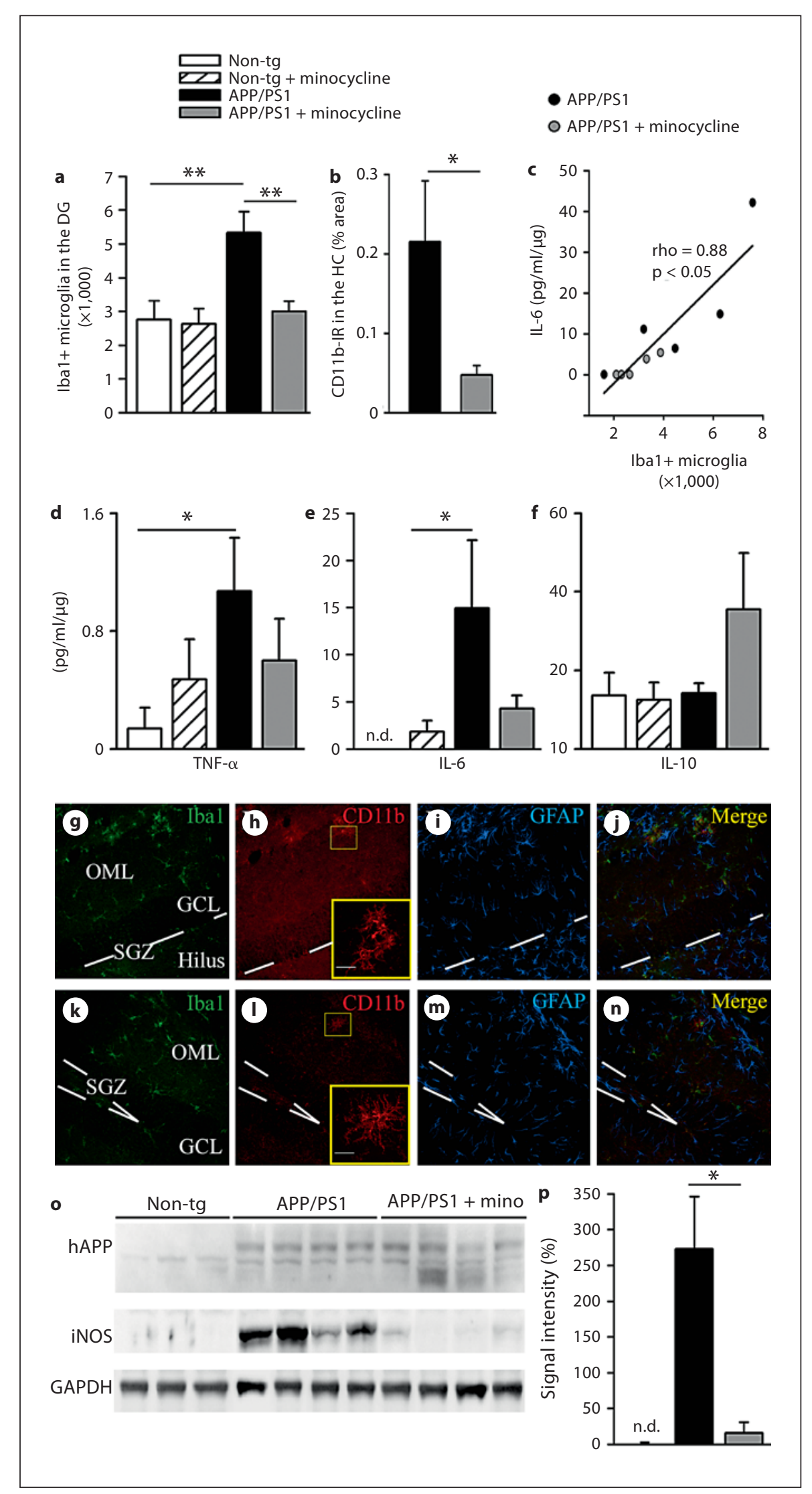



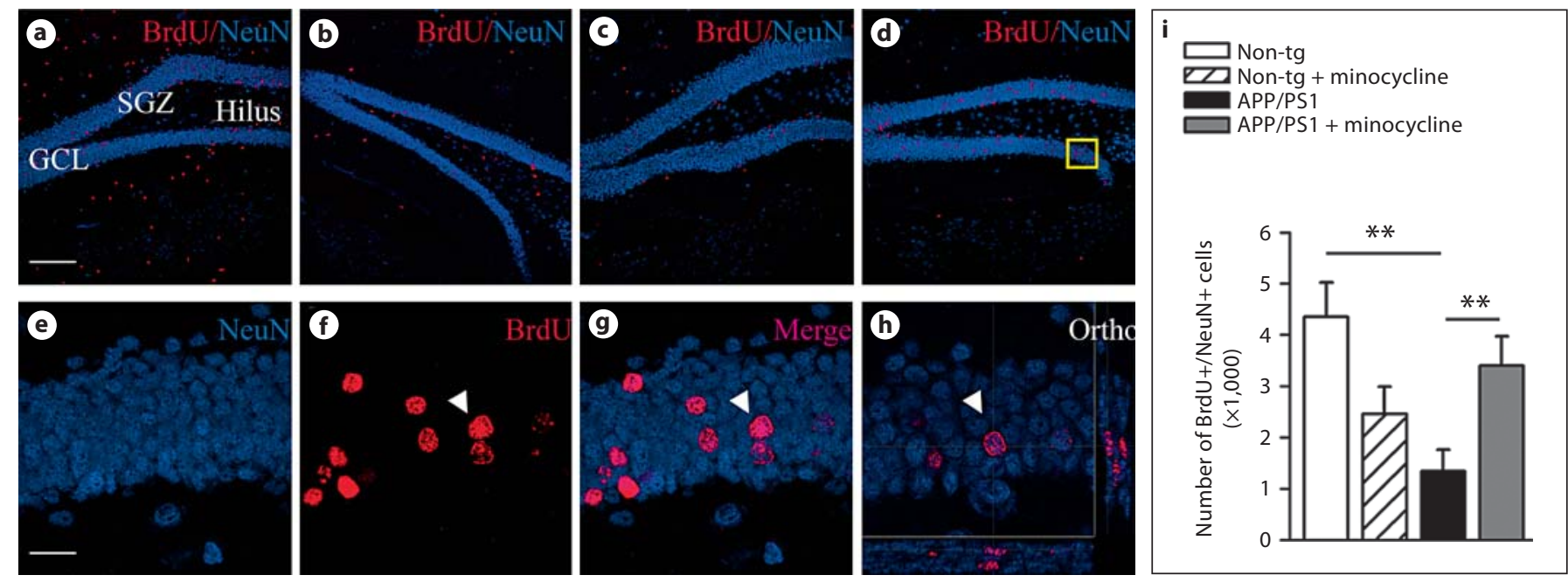

Fig. 2. Minocycline increases numbers of newly-born neurons in APP/PS1 mice. a-d Neurogenesis in the dentate gyrus of non-tg mice: non-tg minocycline treated (a), APP/PS1 vehicle- (b), and minocycline-treated mice (c); detected by double immunofluorescence for NeuN (blue) and BrdU (red) (d). e-h Representative high magnification photomicrographs and orthogonal projection of confocal images of the area depicted by the yellow square in $\mathbf{d}$ confirmed the colocalization of the double-staining BrdU/NeuN. i Minocycline administration resulted in restored numbers of $\mathrm{BrdU}+/ \mathrm{NeuN}+$ new granular neurons. Scale bars $=120 \mu \mathrm{m}(\mathbf{a}), 20$ $\mu \mathrm{m}(\mathbf{e})$. Error bars represent SEM. ${ }^{* *} \mathrm{p}<0.01$ ANOVA followed by Bonferroni post hoc test. mals, and did not differ between minocycline- and vehicle-treated transgenic mice ( $\mathrm{p}=0.58$, online suppl. figure S2A). Also, no significant differences were detected between the groups in numbers of Ki67+ cells ( $p=0.65$, online suppl. figure S2B), DCX+ neuroblasts $(\mathrm{p}=0.64$, online suppl. figure S2C), or PCNA+/DCX+ cells $(\mathrm{p}=0.77$, online suppl. figure S2D).

Taken together, these data provide evidence that minocycline prevented the death of the newly born neurons in APP/PS1 mice without affecting cell proliferation or progenitors' fate choice.

We next verified whether minocycline treatment could affect amyloid levels in the brain of the transgenic animals. The spatial accumulation of $A \beta$ pathology was estimated by the detection of $A \beta$ by ThioS and 6E10. Deposition of $A \beta$ was unaltered among treatment and placebo groups (fig. $3 \mathrm{a}-\mathrm{e}$ ). Measurement of the respective levels of $A \beta_{40}$ and $A \beta_{42}$ in the cortical soluble and insoluble extracts did not reveal any difference among treatment groups (fig. 3f, g). Although not reaching statistical significance, mean levels of soluble $A \beta_{40}$ and insoluble $A \beta_{42}$ were higher in minocycline-treated APP/ PS1 mice ( $\mathrm{p}=0.35$ and 0.25 , respectively). Whether longer anti-inflammatory treatment would have lead to higher levels of $A \beta$, in support of a role for activated microglia in the clearance of A $\beta$ in APP/PS1 mice, awaits further studies. To exclude an effect of minocycline on the different $A \beta$ species, the levels of monomeric $A \beta$ were determined by Western blot assay but no differences among groups were detected (fig. $3 \mathrm{~h}$, i). Monomeric $A \beta$ was also analyzed by Western blot of the insoluble extracts, and no differences were found between groups (data not shown). Together these results show that minocycline promoted the survival of newly born neurons without reducing $A \beta$ pathology and reversing the morphological deficits of newly born neurons associated with $A \beta$-related toxicity.

To analyze the detailed morphology of the new granule cells, we labeled them using hippocampal injection of GFP-expressing retroviral vector and imaged them in a 3-dimensional manner. We found that, despite the increase in the number of newly-born neurons in minocycline-treated mice, the A $\beta$-related morphological deficits were still present during minocycline treatment consistent with the persisting $A \beta$ pathology in the treated mice. Scholl analyses to quantify the extent of dendritic branching at increasing distances from the cell body indicated GFP+ mature new neurons with significantly less dendritic arborization and shorter processes than non-tg equivalents in both vehicle- and minocycline-treated groups (fig. $4 \mathrm{a}, \mathrm{b}$ ). Moreover, the classification of dendritic spines according to the Peters and Kaiserman- 
Fig. 3. Minocycline does not alter $A \beta$ pathology in APP/PS1 mice. a-d Spatial accumulation of $A \beta$ plaques was not affected by minocycline treatment in APP/PS1. Thios staining of compact $A \beta$ and $6 \mathrm{E} 10$ staining of diffuse $A \beta$ did not differ between APP/PS1 vehicle- (a, c) and minocycline-treated mice (b, d). e Quantification of the brain area covered by Thios or 6E10 did not detect any changes, regardless of the treatment. $\mathbf{f}, \mathbf{g}$ Respective concentrations of $A \beta_{40}$ and $A \beta_{42}$ in soluble (f) and insoluble $(\mathbf{g})$ cortical extracts showed no differences among treatment groups. h, i Representative 6E10 immunoblot from soluble cortical homogenates minocycline-administration had no effect on monomeric $A \beta$ species (h), as quantified by densitometric analysis of $A \beta$ signal intensity normalized to $\beta$-actin (i). Error bars represent SEM. Scale bar $=0.9 \mathrm{~mm}(\mathbf{a})$.
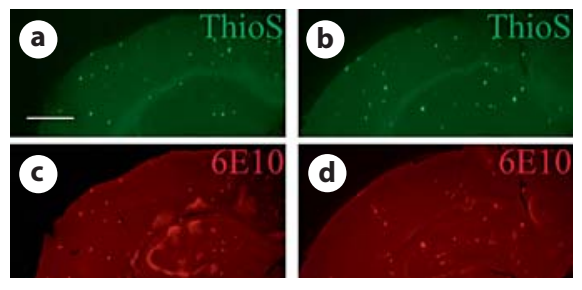

APP/PS1

APP/PS1 + minocycline
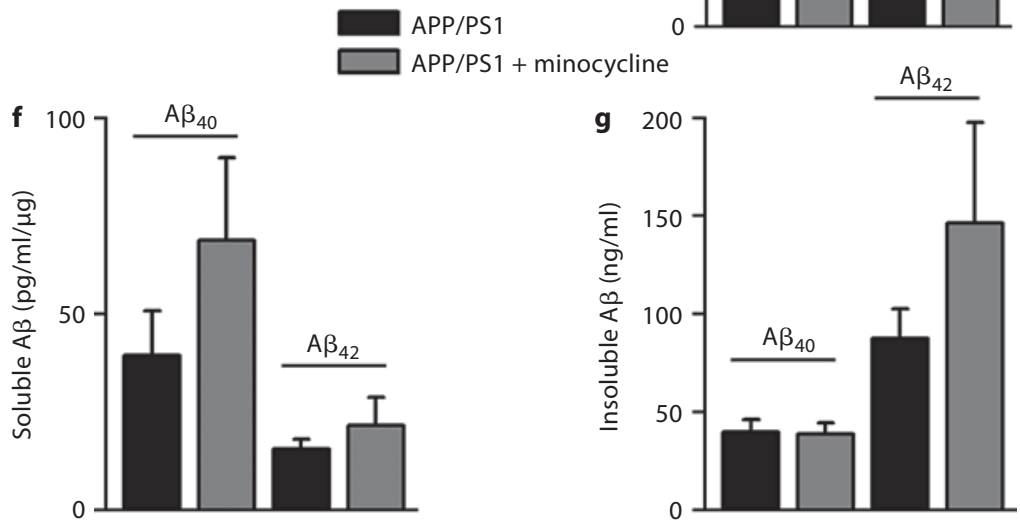

h

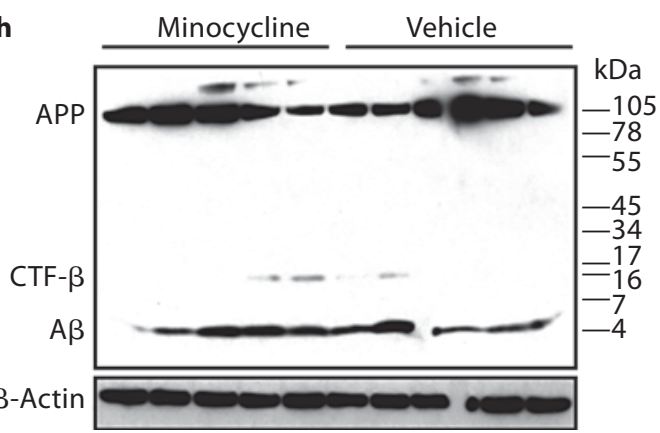

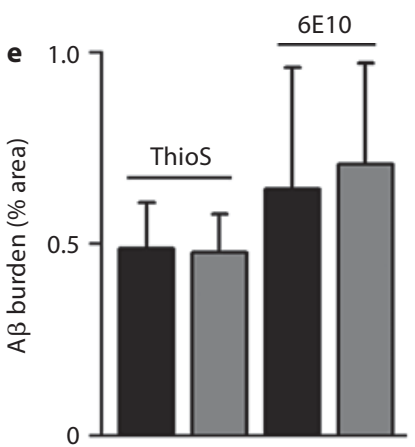

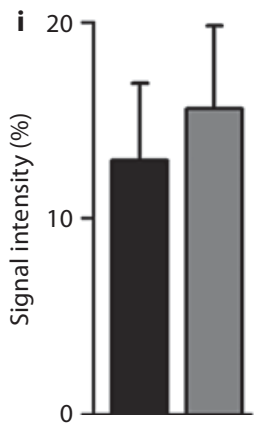

Abramof classification [33] indicated loss of mushroom and stubby spines (fig. $4 \mathrm{c}-\mathrm{e}$ ) but no differences in numbers of long-thin spines (fig. $4 \mathrm{c}, \mathrm{f}$ ).

\section{Minocycline Restores Recognition Memory}

Because minocycline treatment stimulated neurogenesis, we next tested the hypothesis that minocycline could also improve cognitive deficits in APP/PS1 mice. To this aim, we adopted a delayed non-matching-to-sample test, a paradigm based on the tendency of rodents to explore more a novel object than a familiar one [34]. Recognition of the familiar object, and consequent increased interac- tion with the new one, requires intact neural processes mediating storage and/or subsequent recall of the features of the familiar object [23]. Mounting evidence indicates that these neural circuits may benefit from adult neurogenesis [35-38]. We tested all mice after 6-week treatments with minocycline compared to vehicle in a blinded design. Mice were first exposed for $5 \mathrm{~min}$ to an object, the familiar object, followed 20 min later by exposure to a novel object presented together with the familiar object. Vehicle-treated APP/PS1 mice failed to recognize the familiar object, spending less amount of time exploring the novel one, indicating recognition memory im- 
Fig. 4. Minocycline does not rescue dendritic atrophy and loss of spines in APP/ PS1 mice. a Scholl analysis of mature new granular cells labeled with a retroviral vector expressing GFP revealed loss of dendritic complexity in APP/PS1 mice compared to non-tg mice, regardless of the treatment. The graph represents the mean number of intersections between dendrites and concentric radii, centered at the cell body, as a function of distance from the soma, $\mathrm{n}=10$ cells per group. $\mathbf{b}$ Representative confocal pictures of GFP+ mature new neurons for the three groups in study. Computer-assisted classification of spines along $40-\mu \mathrm{m}$ segments did not detect difference in the number of 'long-thin' spines among groups (c); however, the analysis reported lower numbers of 'mushroom' spines (d) and 'stubby' spines (e) in APP/PS1 mice, both vehicle- and minocycline-treated, compared to non-tg $(n=25$ segments per group). f Selected high-magnification segments from respectively non-tg, APP/PS1 vehicle- and minocycline-treated mice. Scale bars $=60 \mu \mathrm{m}(\mathrm{b})$, $5 \mu \mathrm{m}(\mathbf{f})$. Error bars represent SEM. * $\mathrm{p}<$ $0.05,{ }^{*} \mathrm{p}<0.001$, ANOVA followed by Bonferroni post hoc test.

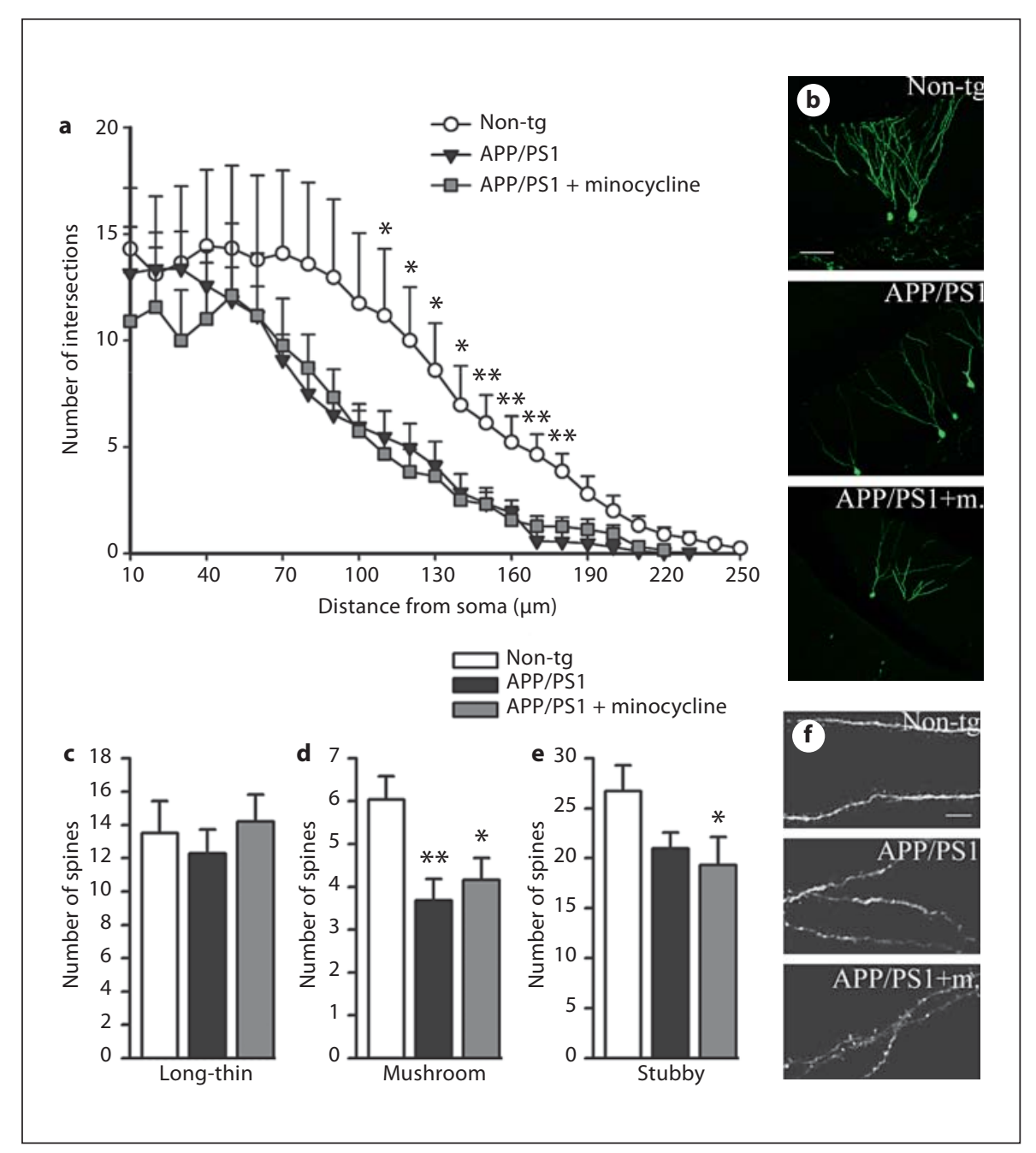

pairment. Minocycline rescued this phenotype to levels of non-tg control mice (fig. 5a). The average times spent interacting with the objects $\mathrm{A}$ and $\mathrm{B}$ during the novelty phase are shown in figure $4 \mathrm{~b}$. To determine whether the mice discriminate between familiar and novel objects, the exploration ratios obtained under each condition were compared with the expected 0.5 chance ratio using one-sample $t$ tests. Non-tg mice, as well as minocyclinetreated APP/PS1, had exploration ratios that were significantly above the chance level $(\mathrm{t}=3.483, \mathrm{p}=0.04$ and $\mathrm{t}=$ $3.945, \mathrm{p}=0.0056$, respectively), whereas vehicle-treated APP/PS1 mice did not differ from chance level $(t=2.055$, $p=0.17$ and $t=-1.689, p=0.1665$, respectively). The treatment with minocycline had no effect on the locomotor activity nor on the number of rearings (data not shown). Also, there was no difference between groups in the Ymaze, a working memory paradigm (non-tg: $59.73 \pm$
$4.79 \%$ vs. non-tg + minocycline: $63.48 \pm 3.25 \%$ vs. APP/ PS1: $65.34 \pm 2.66 \%$ vs. APP/PS1 + minocycline: $64.99 \pm$ $3.36 \%$, percentage of alternations).

\section{Discussion}

The aim of the present study was to determine the effect of microglia inflammation on adult hippocampal neurogenesis in an $\mathrm{AD}$ mouse model with early onset of plaque deposition. We demonstrated that the treatment of APP/PS1 mice with the anti-inflammatory tetracycline derivative minocycline significantly reduced the numbers of microglia in the dentate gyrus as well as the immunoreactivity of activated microglia and the levels of the inflammation-induced protein iNOS. The reduction in microglial activation was accompanied by a significant 
Fig. 5. Minocycline treatment restores hippocampus-dependent memory deficits in APP/PS1 mice as assessed in a recognition memory task. Behavioral testing was done after 6 weeks of daily treatment with minocycline. a Object recognition performance of APP/PS1 minocycline-treated was significantly better than that of APP/ PS1 vehicle-treated and it was comparable to that of non-tg controls. Object recognition index was calculated as the ratio $(T \mathrm{~B}$ $\left.{ }^{*} 100\right) /(T A+T B)$, where $T A$ and $T B$ are the time spent during the second exposure on object A and object B, respectively. b The average TA and TB per group. Error bars represent SEM. ${ }^{*} \mathrm{p}<0.05$, ANOVA followed by Bonferroni post hoc test.

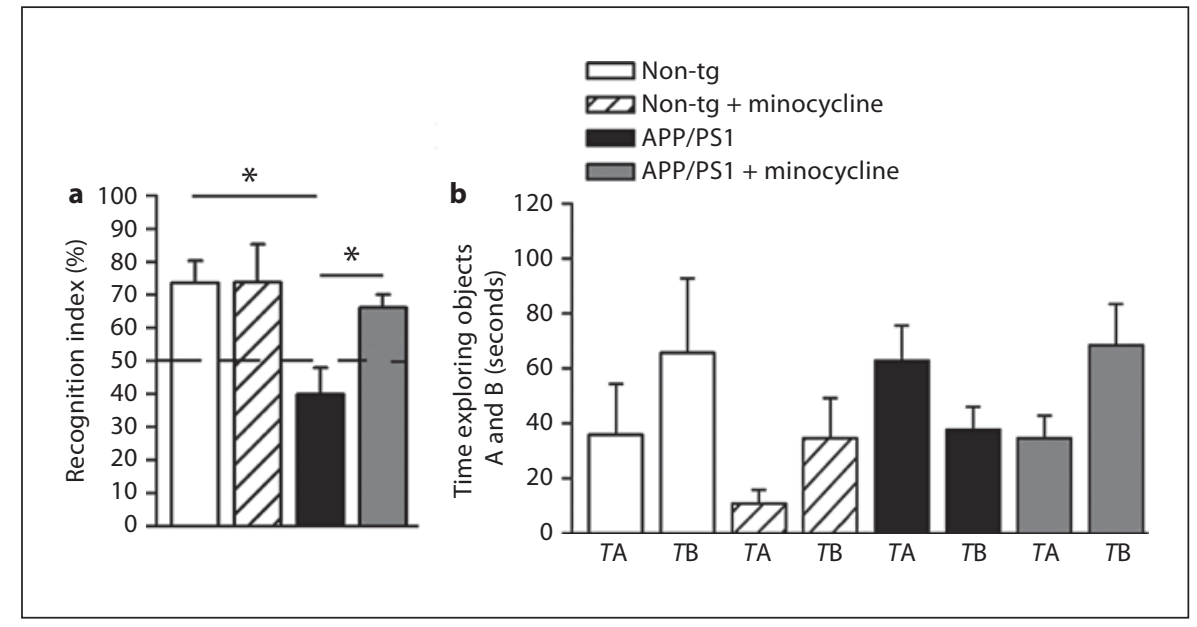

increase in numbers of mature newly formed (BrdU+l $\mathrm{NeuN+}$ ) neurons in the SGZ/GCL, suggesting a protective role for minocycline on the survival of new granule cells. Minocycline neither reduced the $A \beta$ load in the brains of the transgenic mice nor did it restore the morphological deficits and loss of spines in GFP+ new neurons. However, minocycline-treated transgenic mice scored significantly higher in a hippocampus-dependent object recognition test: APP/PS1 without treatment showed a severe impairment in this test, whereas APP/ PS1 mice that received minocycline performed comparably to non-tg mice.

APP/PS1 doubly transgenic mice develop A $\beta$ pathology accompanied by a strong inflammatory response [6]. Inflammation as a result of $A \beta$ pathology may be involved in driving disease progression and neurodegeneration $[5,39]$. Therefore, anti-inflammatory drugs are being tested in clinical trials, however, with contradictory results obtained so far [40]. Consistent with our results, two independent studies with different transgenic mouse models indicated that reduction of microglia activation with minocycline improved cognition in the presence of unchanged $A \beta$ burden $[14,18]$. Our results are in line with these interpretations, and raise the possibility that minocycline-induced rescue of neurogenesis contributes to this effect.

On the other hand, Ziv et al. [41] reported that hippocampal neurogenesis induced by an enriched environment was associated with both the recruitment of $\mathrm{T}$ cells and the activation of microglia, and that it was accompanied by improved spatial learning abilities. Moreover, familial AD-causing PS1 mutations expressed in microglia blocked enriched environment-related hippocampal pro- genitor cell proliferation and differentiation [42]. Together, these and our results suggest that microglia can support neurogenesis in certain conditions while blocking it in others, most likely in dependence on their molecular phenotype. Here we found that minocycline-evoked attenuation of microglia activation, resulting from $A \beta$ pathology, protects neurogenesis. Similar data, noteworthy in the presence of both astro- and micro-gliosis ablation, were reported in another study where vector-mediated overexpression of an anti-inflammatory cytokine in the brain of APP/PS1 bigenic mice resulted in attenuation of AD-like pathology, improved behavior, and increased neurogenesis [43]. In accordance, minocycline changed the cytokine profile in our animals from proinflammatory to anti-inflammatory indicated by decreased TNF $\alpha$ and IL- 6 and possibly increased IL-10 levels [32, 44, 45].

Although not reaching statistical significance, minocycline treatment showed a tendency to reduce adult neurogenesis in non-tg animals (about 40\% less BrdU+l $\mathrm{NeuN}+$ cells than non-tg vehicle-treated littermates). Interestingly, Noble et al. [46] observed a detrimental effect of minocycline on non-tg animals: minocycline decreased tau phosphorylation and aggregation in tau transgenic mice, but increased tau phosphorylation in non-tg mice treated with minocycline. Although this observation awaits further studies, they suggested that different targets of minocycline may be differently affected depending upon the distinctive up-regulation of their pathways. In agreement with a possible dual role of the treatment, minocycline was reported to have different effects at different ages of the same transgenic AD mouse model [18]. These data could also partially explain why, in spite of a plethora of successful trials at the laboratory 
scale, minocycline never succeeded at passing clinical trials [40].

Our results support a detrimental action of proinflammatory microglia on adult hippocampal neurogenesis in transgenic mice expressing familial AD-causing APP and PS1 mutations. We have previously shown that $A \beta$ immunotherapy promotes the survival of newly born neurons in APP/PS1 mice and restores their dendrite branching and spine density [22]. Here, we found that downregulation of neuroinflammation leads to increased survival of new neurons. In contrast, we did not detect any improvements in the morphology or spine numbers of newly born neurons following minocycline treatment. This result may be explained by the unchanged $\mathrm{A} \beta$ burden, known to affect synaptic functions leading to the disruption of the neural networks [47].
Whether the combination of $A \beta$ immunotherapy and anti-inflammatory treatment can result in additive improvement of hippocampal regeneration in $\mathrm{AD}$ mouse models, including the functional integration of the new neurons in the pathological environment [48], awaits further studies.

\section{Acknowledgements}

Supported by SNF grants, the NCCR Neuronal Plasticity and Repair, and the Swedish Research Council. We are grateful to Dr. Irene Knüsel for expert assistance with the behavioral test, to Drs. Ivana Vodopivec, Mario Merlini and Stephanie Trouche for scientific advice and technical assistance, and to Prof. Sebastian Jessberger for fruitful discussions. The authors also thank Prof. Dr. Karen Duff for providing PS1 mice.

\section{References}

$>1$ Haass C, Selkoe DJ: Soluble protein oligo- 11 Lie DC, et al: Neurogenesis in the adult mers in neurodegeneration: lessons from the Alzheimer's amyloid beta-peptide. Nat Rev Mol Cell Biol 2007;8:101-112.

-2 Marcello E, Epis R, Di Luca M: Amyloid flirting with synaptic failure: towards a comprehensive view of Alzheimer's disease pathogenesis. Eur J Pharmacol 2008;585: 109-118.

3 Combs CK, et al: beta-Amyloid stimulation of microglia and monocytes results in TNFalpha-dependent expression of inducible nitric oxide synthase and neuronal apoptosis. J Neurosci 2001;21:1179-1188.

4 Morgan D, et al: Dynamic complexity of the microglial activation response in transgenic models of amyloid deposition: implications for Alzheimer therapeutics. J Neuropathol Exp Neurol 2005;64:743-753.

$>5$ Wyss-Coray T: Inflammation in Alzheimer disease: driving force, bystander or beneficial response? Nat Med 2006;12:1005-1015.

6 Gordon MN, et al: Time course of the development of Alzheimer-like pathology in the doubly transgenic PS1+APP mouse. Exp Neurol 2002;173:183-195.

-7 Grathwohl SA, et al: Formation and maintenance of Alzheimer's disease beta-amyloid plaques in the absence of microglia. Nat Neurosci 2009;12:1361-1363.

$>8$ van Praag $H$, et al: Functional neurogenesis in the adult hippocampus. Nature 2002;415: 1030-1034.

9 Zhao C, Deng W, Gage FH: Mechanisms and functional implications of adult neurogenesis. Cell 2008;132:645-660.

10 Ming GL, Song H: Adult neurogenesis in the mammalian central nervous system. Annu Rev Neurosci 2005;28:223-250. brain: new strategies for central nervous system diseases. Annu Rev Pharmacol Toxicol 2004;44:399-421.

12 Ekdahl CT, Kokaia Z, Lindvall O: Brain inflammation and adult neurogenesis: the dual role of microglia. Neuroscience 2009;158: 1021-1029.

13 Ekdahl CT, et al: Inflammation is detrimental for neurogenesis in adult brain. Proc Natl Acad Sci USA 2003;100:13632-13637.

14 Fan R, et al: Minocycline reduces microglial activation and improves behavioral deficits in a transgenic model of cerebral microvascular amyloid. J Neurosci 2007;27:30573063.

15 Hunter CL, Bachman D, Granholm AC: Minocycline prevents cholinergic loss in a mouse model of Down's syndrome. Ann Neurol 2004;56:675-688.

16 Pattison LR, et al: Apoptotic cascades as possible targets for inhibiting cell death in Huntington's disease. J Neurol 2006;253:11371142 .

17 Quintero EM, et al: Behavioral and morphological effects of minocycline in the 6-hydroxydopamine rat model of Parkinson's disease. Brain Res 2006;1093:198-207.

18 Seabrook TJ, et al: Minocycline affects microglia activation, Abeta deposition, and behavior in APP-tg mice. Glia 2006;53:776782 .

19 Zhu S, et al: Minocycline inhibits cytochrome $c$ release and delays progression of amyotrophic lateral sclerosis in mice. Nature 2002;417:74-78.

20 Hsiao K, et al: Correlative memory deficits, Abeta elevation, and amyloid plaques in transgenic mice. Science 1996;274:99-102.
1 Dolbeare F: Bromodeoxyuridine: a diagnostic tool in biology and medicine. Part II: Oncology, chemotherapy and carcinogenesis. Histochem J 1995;27:923-964.

22 Biscaro B, et al: Abeta immunotherapy protects morphology and survival of adult-born neurons in doubly transgenic APP/PS1 mice. J Neurosci 2009;29:14108-14119.

23 Clark RE, et al: Rats with lesions of the hippocampus are impaired on the delayed nonmatching-to-sample task. Hippocampus 2001;11:176-186.

24 Bravo R, Macdonald-Bravo H: Existence of two populations of cyclin/proliferating cell nuclear antigen during the cell cycle: association with DNA replication sites. J Cell Biol 1987;105:1549-1554

25 Imai Y, et al: A novel gene ibal in the major histocompatibility complex class III region encoding an EF hand protein expressed in a monocytic lineage. Biochem Biophys Res Commun 1996;224:855-862.

26 Schmidt ML, et al: Chemical and immunological heterogeneity of fibrillar amyloid in plaques of Alzheimer's disease and Down's syndrome brains revealed by confocal microscopy. Am J Pathol 1995;147:503-515.

-27 Gundersen HJ, Jensen EB: The efficiency of systematic sampling in stereology and its prediction. J Microsc 1987;147:229-263.

28 West MJ, Slomianka L, Gundersen HJ: Unbiased stereological estimation of the total number of neurons in thesubdivisions of the rat hippocampus using the optical fractionator. Anat Rec 1991;231:482-497. 
29 Thored P, et al: Long-term accumulation of microglia with proneurogenic phenotype concomitant with persistent neurogenesis in adult subventricular zone after stroke. Glia 2009;57:835-849.

30 Holcomb L, et al: Accelerated Alzheimertype phenotype in transgenic mice carrying both mutant amyloid precursor protein and presenilin 1 transgenes. Nat Med 1998;4:97100.

-31 Brown GC: Mechanisms of inflammatory neurodegeneration: iNOS and NADPH oxidase. Biochem Soc Trans 2007;35:1119-1121.

- 32 Monje ML, Toda H, Palmer TD: Inflammatory blockade restores adult hippocampal neurogenesis. Science 2003;302:1760-1765.

-33 Peters A, Kaiserman-Abramof IR: The small pyramidal neuron of the rat cerebral cortex: the perikaryon, dendrites and spines. Am J Anat 1970;127:321-355.

34 Bevins RA, Besheer J: Object recognition in rats and mice: a one-trial non-matching-tosample learning task to study 'recognition memory'. Nat Protoc 2006;1:1306-1311.

35 Dupret D, et al: Spatial relational memory requires hippocampal adult neurogenesis. PLoS ONE 2008;3:e1959.
36 Jessberger S, et al: Dentate gyrus-specific knockdown of adult neurogenesis impairs spatial and object recognition memory in adult rats. Learn Mem 2009;16:147-154.

$>37$ Kee N, et al: Preferential incorporation of adult-generated granule cells into spatial memory networks in the dentate gyrus. Nat Neurosci 2007; 10:355-362.

38 Snyder JS, et al: A role for adult neurogenesis in spatial long-term memory. Neuroscience 2005; 130:843-852.

39 Rogers J, et al: Microglia and inflammatory mechanisms in the clearance of amyloid beta peptide. Glia 2002;40:260-269.

40 Plane JM, et al: Prospects for minocycline neuroprotection. Arch Neurol 2010;67: 1442-1448.

41 Ziv Y, et al: Immune cells contribute to the maintenance of neurogenesis and spatial learning abilities in adulthood. Nat Neurosci 2006;9:268-275.

42 Choi SH, et al: Non-cell-autonomous effects of presenilin 1 variants on enrichment-mediated hippocampal progenitor cell proliferation and differentiation. Neuron 2008;59: 568-580.
43 Kiyota $\mathrm{T}$, et al: CNS expression of anti-inflammatory cytokine interleukin- 4 attenuates Alzheimer's disease-like pathogenesis in APP+PS1 bigenic mice. FASEB J 2010;24: 3093-3102.

44 Liu YP, Lin HI, Tzeng SF: Tumor necrosis factor-alpha and interleukin-18 modulate neuronal cell fate in embryonic neural progenitor culture. Brain Res 2005;1054:152158

45 Vallieres L, et al: Reduced hippocampal neurogenesis in adult transgenic mice with chronic astrocytic production of interleukin-6. J Neurosci 2002;22:486-492.

46 Noble W, et al: Minocycline reduces the development of abnormal tau species in models of Alzheimer's disease. FASEB J 2009;23: 739-750.

47 Palop JJ, Mucke L: Amyloid-beta-induced neuronal dysfunction in Alzheimer's disease: from synapses toward neural networks. Nat Neurosci 2010;13:812-818.

48 Jakubs $\mathrm{K}$, et al: Inflammation regulates functional integration of neurons born in adult brain. J Neurosci 2008;28:1247712488. 